\section{Luminance Contrast - a New Visible Light Technique for Examining Transparent Specimens}

\author{
Jörg Piper \\ Clinic Meduna, Bad Bertich, Germany \\ webmaster@prof-piper.com
}

\section{Introduction}

Transparent specimens are usually examined by dark field, phase contrast and interference contrast light microscopy. In dark field, specimens are illuminated by oblique light beams that come from the periphery of the illuminating apparatus. Therefore, some transparent objects, e.g. unstained native bacteria, are barely visible and fine structures inside them are often not visible. In phase contrast, the discernment of fine detail can be reduced by halo artifacts. The intensity of contrast, i.e. the difference in brightness between the background and specimen, is constant and not variable; it is determined by the specification of the phase ring within the phase contrast lens and dependent on the specific phase differences between the specimen and its surrounding medium. Interference contrast images are free from halo artifacts, but their contrast may be lower than in corresponding phase contrast or dark field images, especially, when transparent specimens are examined in thinlayer preparations. In all the illumination techniques, mentioned above, resolving power is dependent on the wavelength of the visible light spectra and the numerical aperture of the microscope's lens. When the numerical aperture is 1.40 , the resolving power is about $0.20 \mu \mathrm{m}$ when the specimen is illuminated by monochromatic green light (wave length $550 \mathrm{~nm}$ fig. 1). This value can be regarded as the usual limit of light microscopic resolution (4). Submicroscopic structures, smaller than $0.20 \mu \mathrm{m}$, can only be recognized in fluorescence microscopy when a light microscope is used.

Recently, luminance contrast has been developed by the author as a new illumination technique characterized by several advantages in comparison with the usual examination methods suitable for transparent specimens. With the help of luminance contrast, such speci-

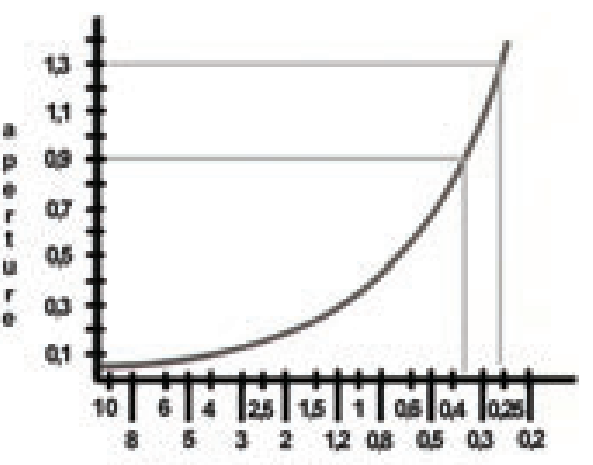

Iateral resolution ( $\mu \mathrm{m})$

Fig. 1 (left): Resolving power [ $\mu \mathrm{m}]$ and numerical aperture (NA) (modified from Leitz - 4)

Fig. 2 (center): Achromatic objective 45/0.65, modified for luminance contrast, plane-parallel plate with centered beam stop in the back focal plane (arrow), simplified light path for luminance dark field. ad = aperture diaphragm, ss = specimen slide

Fig. 3 (right): Light path in mirror objectives (schematic). ad = aperture diaphragm, ss = specimen slide, cs = cover slip. a: bright field. b: luminance dark field. c: luminance phase contrast d: luminance phase contrast, using a special double diaphragm (centric perforation and peripheral light ring)

mens can be examined in several variations of contrast effects, which are similar to dark field, phase and interference contrast. Therefore, these variants have been named luminance dark field, luminance phase contrast and luminance interference contrast. As opposed to common dark field imaging, internal structures within the specimen are visible in high contrast. As opposed to common phase and interference contrast, the intensity of contrast is adjustable in tiny steps; and halo artifacts are either reduced or do not exist. The light path is completely different from common dark field, phase and interference contrast. In all variants of luminance contrast, the specimen appears as a self-luminous, florescent object resulting in fundamental improvement in resolving power.

\section{Principles of luminance contrast}

In luminance contrast, the specimen is illuminated by a very small axial light beam that is congruent with the optical axis. Suitable light beams can be achieved, when the aperture diaphragm of a common bright field condenser and the field diaphragm for Köhler-illumination are appropriately closed. Inside of the objective, preferably within the back focal plane, a small circular light absorbing light-stop has to be situated centrically, congruent with the optical axis (fig. 2). The area of the light stop should be about 10 or 20 percent of the internal cross section area of the objective. The surface of this stop should be a non reflecting black color. By this optical modification, the light path within the objective changes radically. When the illuminating central beam is completely blocked by the light stop, it no longer contributes to the microscope image. Nevertheless, the specimen remains illuminated centrically by this beam. When the intensity of the illuminating light is adequate, the specimen is visible in a maximized homogeneous contrast, situated in a dark black background (luminance dark field). This image is a result of scattered light components bent and reflected by the specimen. The scattered beams can pass all lenses of the objective, because their optical pathway is different from the optical axis and the central illuminating beam and are not blocked by the beam stop. A simplistic diagram of the light path in luminance dark field is shown in fig. 2.

Now, the aperture diaphragm in the condenser can be opened in tiny steps so that a small peripheral component of the central light beam is no longer covered by the light stop and can pass the objective together with the scattered beams emitted by the specimen. A moderate brightening of the background results from this. Both light components (scattered light from the specimen and background light from the periphery of the central beam) interfere with each other. Phase differences within the specimen and its surroundings acquire contrast similar to negative phase contrast (luminance phase contrast). The brightness of the background and the intensity of the contrast can be controlled and regulated by the aperture diaphragm. Existing phase differences remain

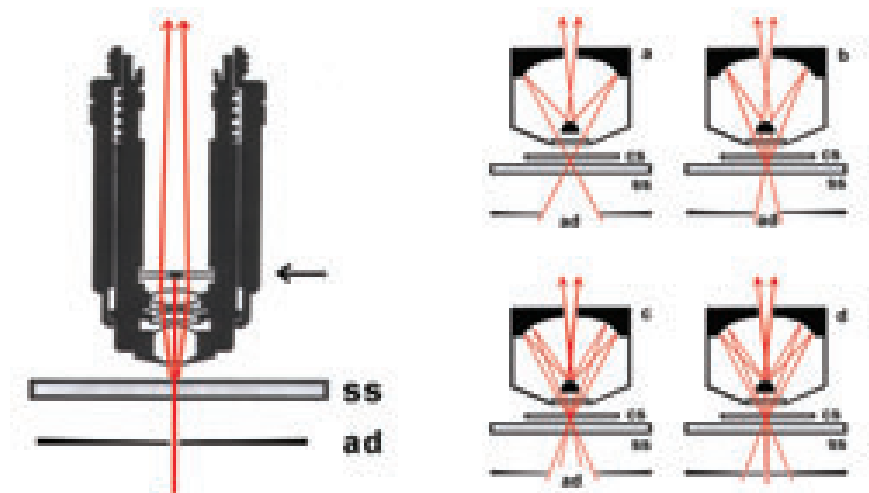

visible, as long as the brightness of the background remains lower than the brightness of the specimen. Otherwise, the illumination changes to bright field.

When the specimen occurs in luminance phase contrast, a part of the illuminating beam can be covered by a non transparent mask shifted into the condenser from one side, situated near the aperture diaphragm. In this way, oblique illumination is achievable so that the structure of the specimen is visible in a three dimensional manner comparable with interference contrast. As described above, the brightness of the background and the intensity of the resulting contrast can be regulated via the aperture diaphragm.

The effects of luminance interference contrast are also achievable without using an additional mask when the light stop is slightly uncentered in the back focal plane of the objective. In this case, an oblique 


\section{THE BIONIFICANT OOMBINATION e EE MORE}

\section{ZEIGG ULTRA}

Ultra high resolution FESEMs for simultaneous BSE and SE real-time imaging together with significant ease of operation. The perfect tool for nano-scale compositional analysis.
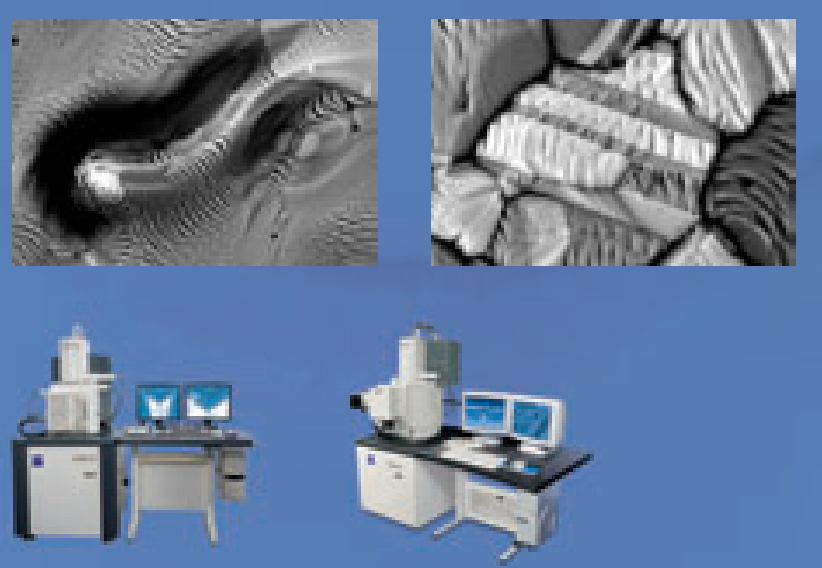

\section{Carl Zeiss SMT Inc}

One Corporation Way

Peabody, MA 01960 USA 


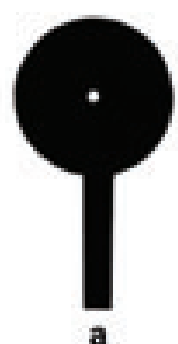

a
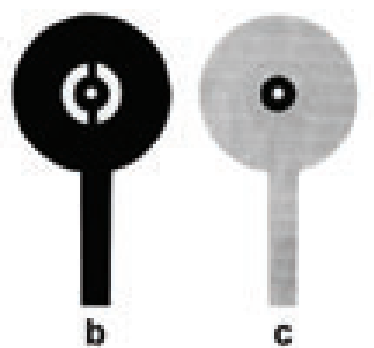

c
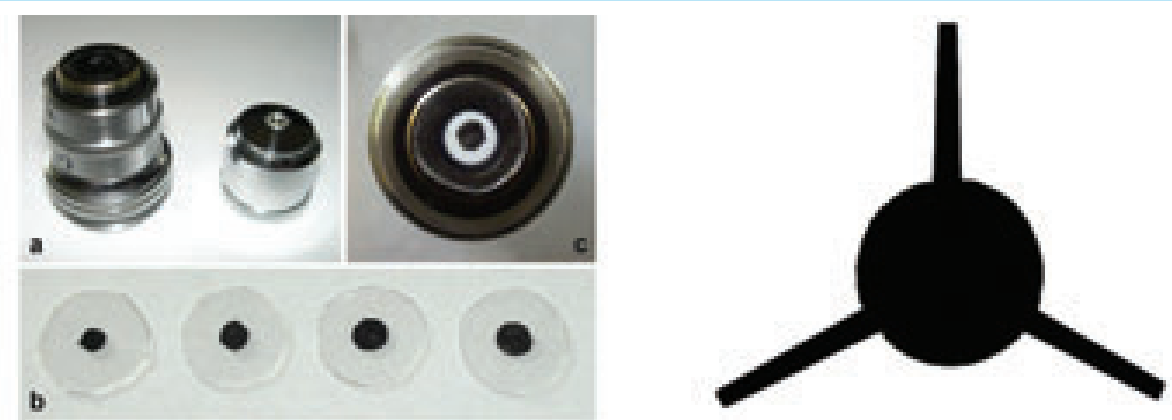

Fig. 4 (left): Models of condenser slides for luminance contrast. a: perforated screen (one centered hole). b: double perforated screen (centric hole and peripher light annulus). $c$ : transparent slide with concentric perforation and marginal boundary arc.

Fig. 5 (center): Rebuilding of the objective 45/0.65 for luminance contrast, a: unscrewing of the objective. b: plane-parallel plates with beam stops in different diameters. $c$ : shaft with absorbing element

Fig. 6 (right): Light absorber with three radial arms (schematic)

illumination occurs as when the aperture diaphragm is in the centered position and closed adequately. When a phase contrast condenser is available instead of a bright field condenser, luminance dark field can also be achieved by producing the centered illuminating light beam with the suitable ring shaped mask. In this case, a small condenser annulus is necessary so that the passing light beams are completely covered by the light stop in the objective; in this technical variant, the condenser aperture diaphragm remains wide open.

Further optimizations can result from several technical modifications with regard to the illuminating apparatus and the objective. When mirror objectives are available, constructed according to the CassegrainSchwarzschild-type $(1,2)$, the backside of the centric convex mirror in the middle of the objective can act as a light absorbing element. In this case, specific advantages of mirror systems are also relevant for high optical quality in luminance contrast. Thus, images are completely free from any chromatic aberration and great visual fields are illuminated homogeneously without any relevant spherical aberration. Fig. 3 demonstrates the optical principles of a mirror objective and the various variants of luminance contrast with regard to the specific light path in these systems. Some technical modifications can be realized, related to the illuminating apparatus. Thus, various condenser slides for luminance contrast can be created (fig. 4). First, a perforated screen can be made (fig. 4a), that can be shifted into the condenser in different ways. The diameter of the perforation has to be adapted to the size of the light stop within the objective so that both elements are optically congruent. When the perforated screen is centered properly, luminance dark field results. When the slide is moderately shifted in an uncentered position, luminance interference contrast can occur. Using this perforated screen, the condenser aperture diaphragm can remain in a wide position or closed moderately if suitable for improved quality.

A double perforated screen can be used, consisting of one centric perforation in the middle and one peripheral annular perforation. Both perforations are separated from each other by an opaque boundary arc (fig. 4b). Alternatively, a transparent slide can be fitted with a centric
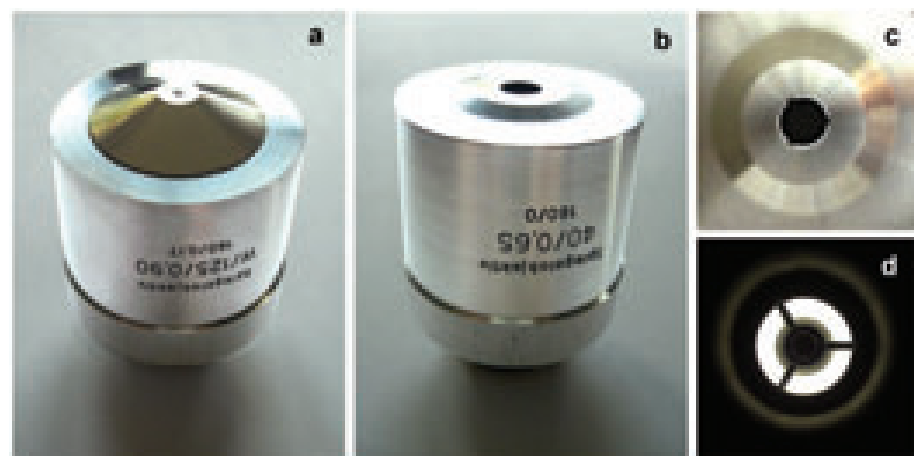

Fig. 7: Mirror objectives Zeiss Jena. a: W 120/0.90 160/0.17 (katadioptric water immersion). b-d: 40/0.65 160/0 (katoptric system) perforation and a marginal boundary arc (fig. 4c). In both variants, the existing separate light corridors for the central and peripheral beams can be filtered in different colors. Using these screens, the intensity of the background light can be adjusted and reduced adequately when the condenser aperture diaphragm is partially closed. By these means, the centric illuminating beams and the peripheral background beams can be separated from each other more rigorously as well in luminance phase contrast as in luminance interference contrast. Moreover, additional color contrast effects can occur, when specimen and background are illuminated in different colors. In a similar manner, color double-contrast can be achieved in luminance dark field when the central illuminating light beam is divided into two parts with different colors.

\section{Material and methods}

A set of three achromatic bright field objectives (Leitz 10/0,25, $45 / 0,65$, oil 100/1,30) were modified for luminance contrast as follows: The $45 \mathrm{x}$ and 100x magnifying objectives were equipped with transparent plane-parallel plates, mounted in the back focal plane of these objectives. In the middle of each plate, a circular beam stop was mounted. The beam stops were in black color, their surfaces matt and non reflecting. By preparing absorbing elements with different diameters, suitable sizes of absorbers could be tested out for each objective. Fig. 5 demonstrates the rebuilding process for the $45 \mathrm{x}$ objective. The $10 \mathrm{x}$ magnifying objective was equipped with a different beam stop, consisting of a black centric area and three radial arms, being free from any transparent reflecting components (construction plan in fig. 6). This element was mounted in an uncentered position so that luminance interference contrast was achievable independent from additional light absorbing slides inside the condenser. Moreover, two Cassegrain-Schwarzschild mirror objectives were tested out, made by Zeiss Jena (fig. 7): 125/0.90 (katadioptric water immersion, suitable for cover slip preparations) and 40/0.65 (katoptric system, only suitable for preparations without cover slips).

All alignments of the beam stops in the objectives and their corresponding illuminating components in the condenser were controlled and documented by a phase telescope. Basic alignments for common objectives with lenses are shown in fig. 8, equivalent alignments for mirror objectives in fig. 9. The microscopic images presented in this article were taken in bulb or flash light, using an Olympus camedia C 7070 camera, adapted with a Leica vario photo ocular (range of magnification: $5 \mathrm{x}-12.5 \mathrm{x})$.

\section{Results}

The $125 \mathrm{x}$ magnifying water immersion leads to excellent results when small transparent specimen are examined in thin-layer preparations (fig. 15-23). This mirror objective can be successfully combined with photo oculars up to $12.5 \mathrm{x}$ magnification. The focal depth is dependent on the respective magnification (maximum here: $125 \times 12.5$ $=1562 \mathrm{x}$ ) and the numerical aperture (here: 0.90). As demonstrated in fig. 10, the focal depth is about $0.8 \mu \mathrm{m}$ when the magnification is $1600 \mathrm{x}$; it increases up to $1.0 \mu \mathrm{m}$ when the magnification is reduced to 


\section{The Power of SEMI Ultra-high Resolution SEM/STEM}

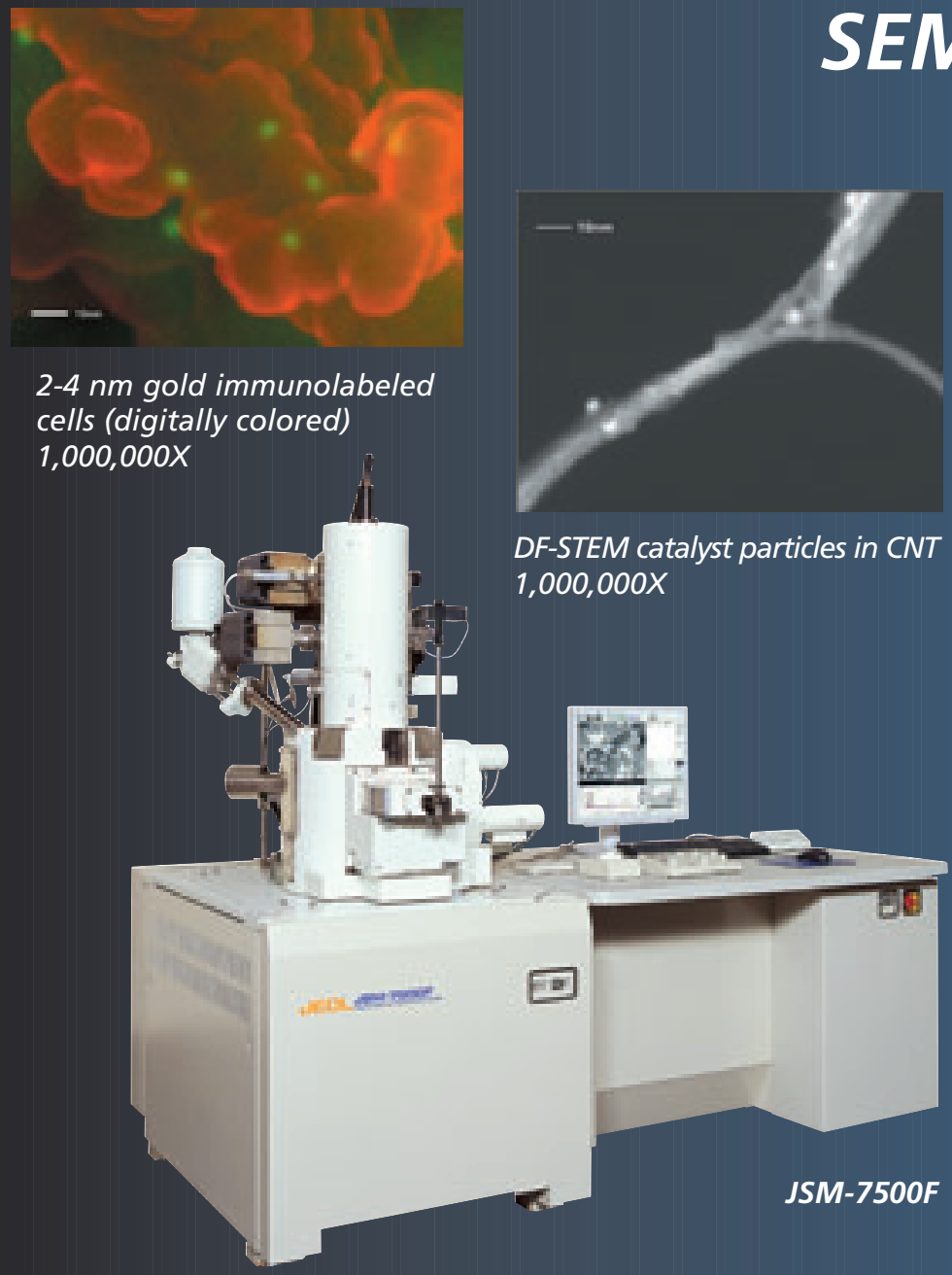

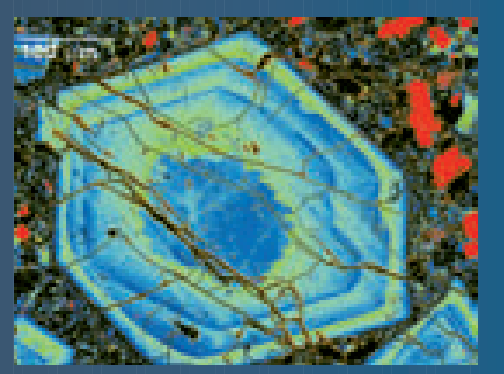

Zoned feldspar (digitally colored)

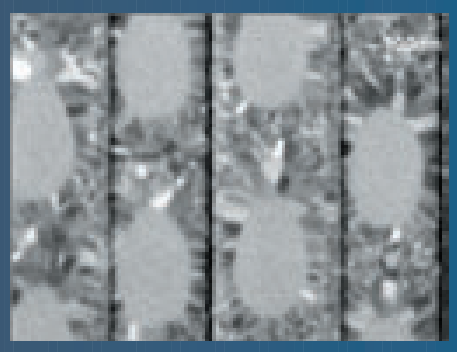

$0.8 \mathrm{kV} L A B \mathrm{e}^{*}$ image of DVD surface
Unlock the true power of the new cold cathode FE SEM from JEOL. Examine nanostructure details with STEM and SEM flexibility, true $1,000,000 \mathrm{X}$ imaging, high beam currents, and the most sensitive low-angle backscatter detector in the industry. An ultra-stable stage with full range of motion in all axes eliminates image shift at the extreme nanoscale.
Expect more from your high performance SEM. With 50+ years of SEM leadership and award-winning service, JEOL can give you unbeatable imaging and applications support.
Seeing is believing - call us for a demo today, or visit wWw.jeolusa.com/powerSEM.
Another Extreme Imaging Solution

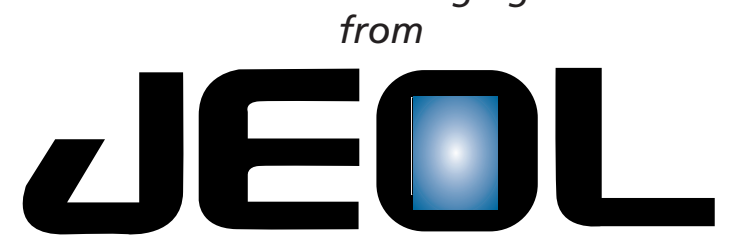

Stability • Performance • Productivity jeolusa.com • salesinfo@jeol.com 

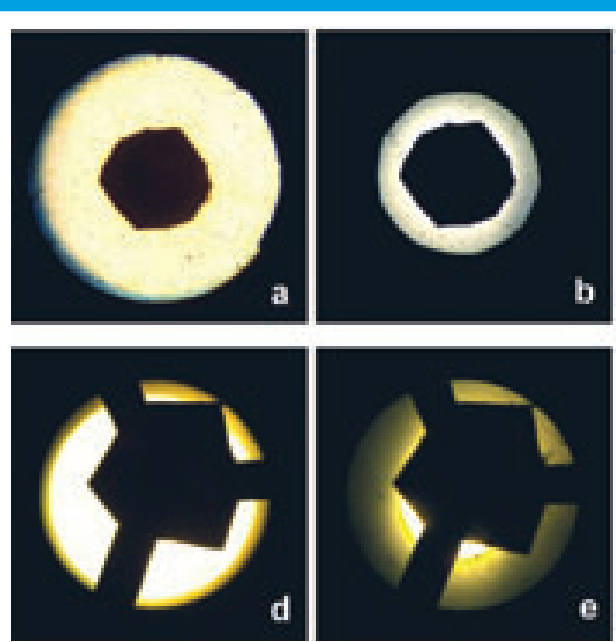

Fig. 8: Typical alignments in luminance contrast objectives (modified lenses), controlled by a phase telescope. a: objective 45/0.65, bright field. $b$ : objective $45 / 0.65$, luminance phase contrast. c: objective $45 / 0.65$, luminance dark field, d: objective 10/0.25, bright field. e: objective 10/0.25, luminance interference contrast. f: objective 10/0.25, luminance dark field
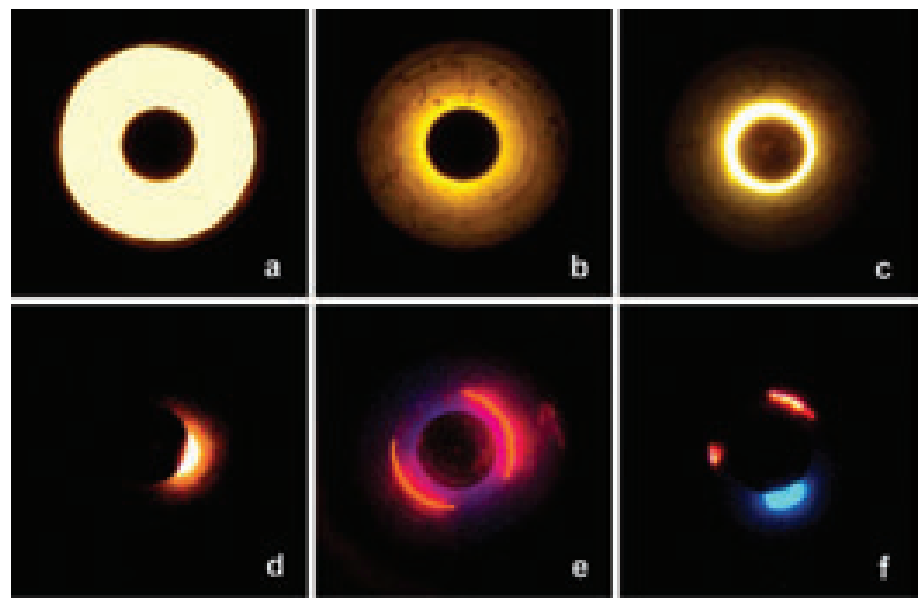

Fig. 9: Typical alignments in mirror objectives, controlled by a phase telescope. a: bright field. $b$ : luminance dark field. c: luminance phase contrast. d: luminance interference contrast (uncentered perforated screen in the condenser). e: luminance phase contrast with two-colors double-contrast. f: luminance interference contrast with two-colors double contrast

$1000 \mathrm{x}$. These values for focal depth are about $66-78 \%$ higher than the corresponding values calculated for a conventional oil immersion lens with a numerical aperture of 1.30 (3). The specimen can be examined and photographed easily, because the focal depth of this mirror objective is above average. The lateral resolution is determined by the numerical aperture (fig. 1). When the aperture is 0.9 , the resolution is about $0.38 \mu \mathrm{m}$. When the aperture increases up to 1.30 , the resolution is near $0.30 \mu \mathrm{m}(4)$. Thus, the lateral resolution of this mirror objective is reduced by about $27 \%$ when compared with a common oil immersion lens. In practice, this moderate reduction of the calculated resolving power is compensated for by the specific illumination effects of luminance contrast (further explanations in the discussion).

The contours of cell membranes and cell walls, including their superficial structures, are seen in extraordinary clarity. Discontinuances of these structures, for example pores or perforations in nucleus membranes or cell walls can be detected accurately as well as various intracellular structures like photoreceptors, plastides, vaculoes,
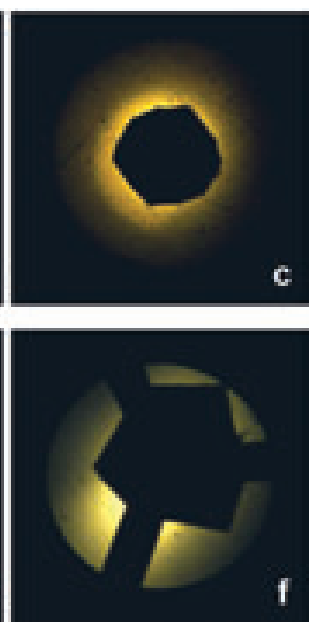

and ejectiosomes etc.. Also fine nuances in the cytoplasm of bacteria and protozoa are visible, especially in luminance dark field. The 40x magnifying mirror objective is suitable for examination of fine structures in crystallizations and other particles in preparations without coverslips. The color filters shown in fig. $4 \mathrm{~b}$ and $\mathrm{c}$ can also be used successfully for bicolor double-contrast techniques in colorless specimen (fig. 14).

All images taken with mirror objectives are characterized by a planarity and a complete absence of artifactual reflections or vignetting as well as high saturation and clarity of colors; the faithfulness of color detection is higher than

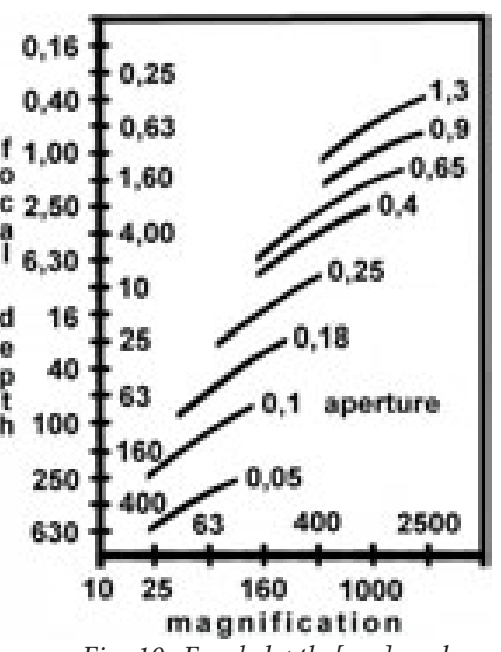

Fig. 10: Focal depth [um] and total magnification, nomograms for several numerical apertures (modified from Determann and Lepusch - 3) in apochromatic lenses. When modified objectives with glass lenses are used, all variants of luminance contrast can be achieved. As the respective light beam stops are situated in the back focal plane, they are not visible in microscopic images. In all variants of luminance contrast, the $10 \mathrm{x}$ magnifying lens is free from any reflections or vignetting (fig. 1113). Bicolor double-contrast can be achieved in the same manner using higher magnifying mirror objectives (fig. 13). All variants of luminance contrast can also be carried out in polarized light, when anisotropic materials, e.g. chitin structures are to be examined (fig. 11).

When carried out using the 45 and $100 \mathrm{x}$ magnifying lenses, luminance contrast leads to extraordinary visible enhancements of optical resolving power when compared with conventional illuminating techniques (fig. 24-26). For example, marginal structures in bacteria walls are visible in images taken with the $45 \mathrm{x}$ lens that are smaller than the usual limit of light microscopic resolving power. Using the $100 \mathrm{x}$ magnifying oil immersion, fine textures on the surfaces of epithelial cells and villous structures on their marginal cell membranes can be recognized in a similar manner when using the $125 \mathrm{x}$ magnifying mirror objective. The locally manufactured prototypes of these lenses and the mounted plane-parallel plates are not optimally coated. Therefore, some reflections occur in luminance dark field, when the field diaphragm is not appropriately closed. When this diaphragm is properly closed most of these artifacts can be greatly reduced so that corresponding photographs are free from visible artifacts.

\section{Further technical developments}

Special condensers for luminance contrast illumination could be
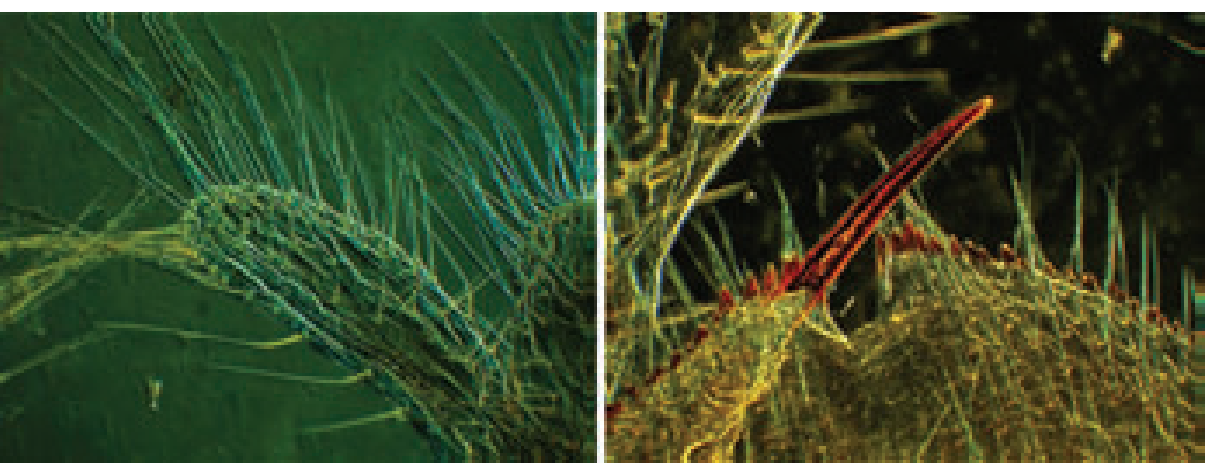

Fig. 11 (left): Jaw of a dragonfly larva, objective 10x, ocular $5 x$, luminance interference conrast, polarized light, horizontal field width (HFW): $1600 \mu \mathrm{m}$

Fig. 12 (right): Data from fig. 11, luminance dark field 

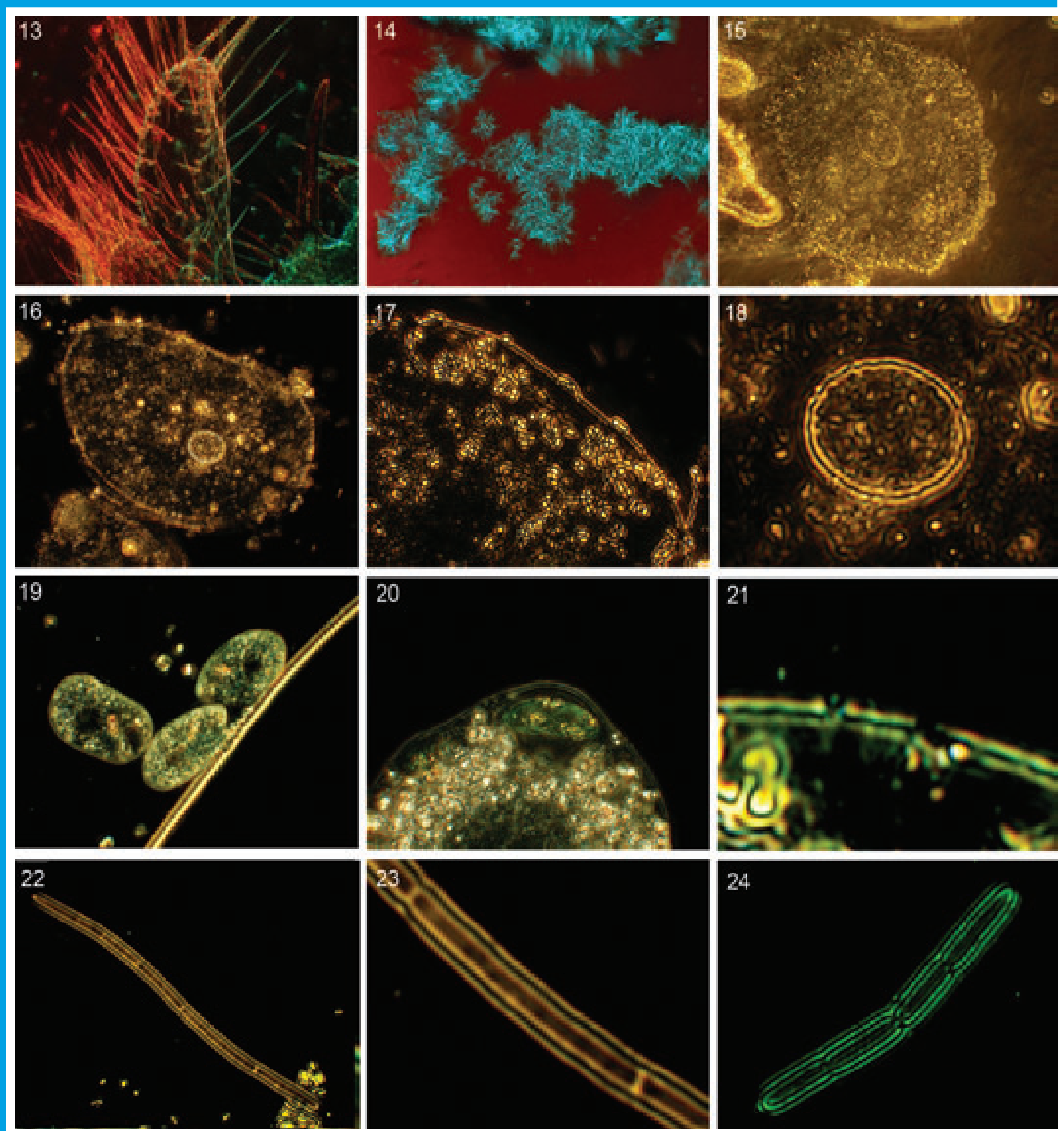

Fig. 13: Data from fig.11, luminance dark field with two-colors double contrast (photograph taken by Timm Piper)

Fig. 14: Sodium-chloride, thin-layer crystallization, mirror objective 40x, ocluar 8x, luminance phase contrast in two-colors double contrast, HFW: 200 um Fig. 15: Epithelial cell, mirror objective WI 125x, ocular 6x, luminance interference contrast, HFW: $100 \mu \mathrm{m}$

Fig. 16: Epithelial cell, mirror objective WI 125x, ocular 6x, luminance dark field, HFW: $100 \mu \mathrm{m}$

Fig. 17: Epithelial cell, mirror objective WI 125x, ocular 12,5x, luminance dark field, cell membrane with sessile bacteria, HFW: $50 \mu m$

Fig. 18: Epithelial cell, mirror objective WI 125x, ocular 12,5x, luminance phase contrast, adjusted for high contrast intensity, nucleus, pores in the nuclear membrane, HFW: $25 \mu \mathrm{m}$

Fig. 19: Flagellates (chilomonas) near the marginal zone of a small aerocyst,mirror objective WI 125x, ocular $6 x$, luminance dark field, HFW: $100 \mu$ m

Fig. 20: Small ameba (actinophrys), ectoplasmatic vacuole with food particle, mirror objective WI 125x, ocular 12.5x, luminance dark field, HFW: 50 um

Fig. 21: Perforations in the cell wall of a flagellate (euglena), mirror objective WI 125x, ocular 12.5x, luminance dark field, HFW: $6 \mu \mathrm{m}$

Fig. 22: Dental bacillus, mirror objective WI 125x, ocular 12.5x, luminance dark field, HFW: $60 \mu \mathrm{m}$

Fig. 23: Detail from fig. 21, visible differences in local cytoplasmatic density, HFW: $20 \mu \mathrm{m}$

Fig. 24: Dental bacillus, objective 45x, ocular 12.5x, luminance dark field, HFW: $30 \mu \mathrm{m}$ 
developed so that the geometry of the illuminating light beams (central and peripheral components) could be adapted more optimal to the geometry of the beam stops within the objectives and the optical characteristics of the respective specimen. Thus, individual sets of double diaphragms could be made for special sets of corresponding objectives. These light modulating elements could be created as slides or modified filters that can be shifted into the condenser. Alternatively, a condenser for luminance contrast could be constructed as an universal condenser, equipped with several removable turrets. In this case, one special turret could be built up for luminance contrast, containing a suitable set of different diaphragms. Fig. 27a shows the construction principle of a double diaphragm for this purpose. Other turrets might be made for conventional illuminating techniques (e.g. phase contrast and dark field) in the usual manner, so that the microscopist could turn from luminance contrast to phase contrast by changing the turrets.

Moreover, modified condensers could be equipped with special double diaphragms consisting of one or two iris diaphragms (fig. 27 b-d). The dimensions of the central perforation and the peripheral light ring could be regulated in tiny steps independent of each other. Additional optimizing effects might result from this. As demonstrated by the hand-made prototypes, shown in fig. 4 b and c, double-contrast color effects could be achieved, when the double diaphragms were fabricated as two-colored filters. Pairs of two complementary colors might lead to best contrasting results. To achieve three dimensional effects, all modified condensers, described above, could be equipped additionally with non transparent masks as mentioned in the section about principles of luminance contrast. Alternatively, the light modulating elements in the condenser itself or the light beam stop in the objective could be off centered moderately.

Because of its high contrast and extraordinary resolving power, luminance contrast should also be appropriate for applications when combined with fluorescence illumination techniques. When epiflourescence is used, luminance contrast could be superimposed with fluores-
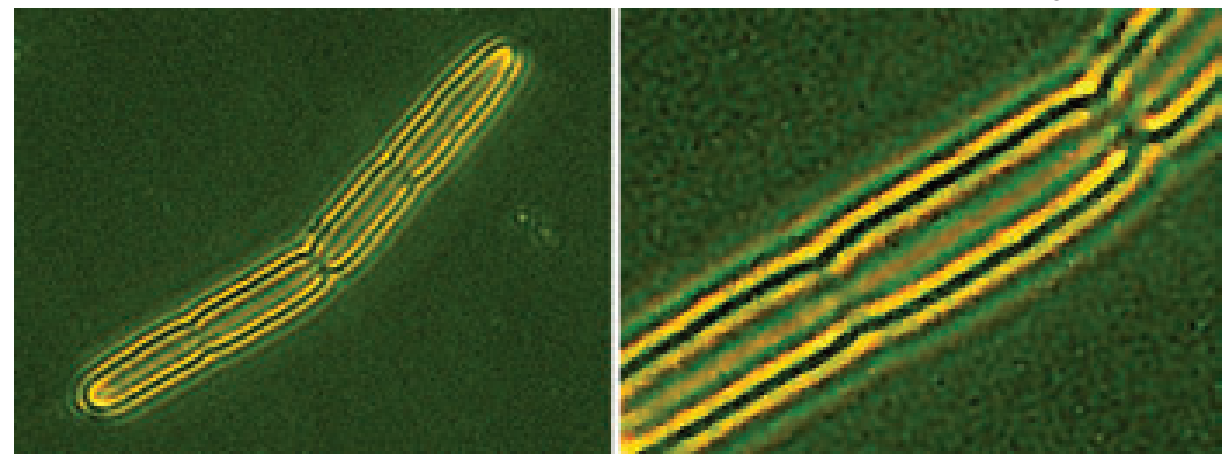

Fig. 25 (left): Data from fig. 23, luminance phase contrast

Fig. 26: Detail from fig. 24, marginal line breadth of the bacteria wall: ca $0.20 \mu \mathrm{m}, \mathrm{HFW}: 10 \mu \mathrm{m}$ the peripheral light annulus within the condenser. In this manner, the intensity of the luminance contrast image and the energy of the excitation light can be independently regulated. In all technical variants, especially luminance dark field, we obtain extraordinary results when combined with fluorescence.

\section{Further modifications of light absorbing components}

When modified objectives containing glass lenses are used for luminance contrast, the central position of the light absorbing beam stop necessary for this could be regarded as a potential disadvantage, because the axial light components do not contribute to the image. In this theoretical respect, other technical modifications might be an advantage. Thus, the central beam stop could be replaced by a non transparent annular shaped light absorber, fitted in the lenses like a phase ring in phase contrast objectives. In the same way as in phase contrast microscopy the condenser can be fitted out with light rings. When the light beams passing through the light ring within the condenser are completely covered by the annular shaped absorber within the objective, the specimen will be illuminated in luminance dark field. Fig. 28a shows the light path of this arrangement and the corresponding light ring in a conventional phase contrast condenser. When the light ring within the condenser is modified to a small transparent sectional gap, the illuminating light comes from only one direction and runs to the specimen in an oblique way. Additional relief effects will result from this (fig. 28b). Luminance phase contrast can be achieved, when the light ring and the annular shaped light absorber are moderately misaligned, so that a small part of the illuminating light is not absorbed within the objective.

Moreover, condensers can be modified to achieve light rings in variable breadths. For this purpose, a centered, circular-shaped light absorbing plate can be mounted in the condenser working in cooperation with the aperture diaphragm (fig. 29a). When a condenser for bright field is used, a transparent filter with a centered light absorber (fig. 29b) can be shifted into the condenser near the plane of the aperture diaphragm. When the light absorbing plate is centered, it can be properly aligned with the aperture diaphragm. The breadth of the light ring can be regulated by the width of the aperture iris diaphragm (fig. 29 c-e). To achieve luminance contrast effects, the margin of the light absorber within the condenser has to be in optical congruence with the inner diameter of the light absorbing annulus in the corresponding objective. In this manner, luminance dark field will result when the condenser iris diaphragm is adequately closed. Luminance phase contrast will occur, when the iris diaphragm is moderately opened. Luminance interference contrast could be achieved, when the light beams are partially covered inside the condenser.

cence microscopic images in the same manner as phase or interference contrast images are usually combined with epiflourescence. When luminance contrast is combined with fluorescence in transmitted light, two different technical considerations should be taken into account. First, the excitation light for fluorescence illumination and the lower intense illuminating light for luminance contrast must be directed to the specimen via the same pathway through the central condenser diaphragm. In this case, both light sources are combined with each other, running within the central beam. The illuminating light for luminance contrast should preferably be monochromatic; the wave length of this light should be different from the wavelengths related with the fluorescence image. Secondly, both components of illuminating light could be separated from each other within the illuminating apparatus. To achieve this, only the central light for luminance contrast should pass through the central diaphragm, so that the central beam should just contribute to the luminance contrast image. The excitation beam should run through
Likewise, universal condensers for phase contrast microscopy equipped with turrets might be modified for variable luminance contrast effects, when their usual fixed light rings are replaced by a set of circular light absorbers with different diameters, adjusted to the geometry of the annular light absorbers within the corresponding luminance contrast lenses. The breadth of the respective light ring could be regulated by the aperture iris diaphragm as described above. Despite these theoretical considerations, severe problems can occur in practice, when annular light absorbers are fitted in lenses instead of small circular beam stops in centered positions. According to our practical evaluations and first experiences with this, the resulting quality of microscopic images is worse, although the central light beams are no longer influenced by this technical modification. It could be taken into account as a reason for this that the total area of an annular light absorber is mostly higher than the area of a small centric beam stop in the middle of an objective (higher "missing disk"). Loss of contrast and sharpness can result. 


\section{SiriusSD}

\section{Silicon drift detector for EDS applications}

SiriusSD is a silicon drift detector designed to make short work of X-ray analysis. Its industry-standard restored preamplifier output provides some compelling benefits.
Contact us today for information on how you can upgrade your EDS system to include a SiriusSD detector, or for details of system suppliers offering SiriusSD.

Consider the SiriusSD advantage:

- No liquid nitrogen

- Analytical quality performance

- High rate capability

- Excellent resolution

- Stable peak position and resolution over a broad range of count rates

- Flexible system integration

See us at Microscopy and Microanalysis 2007 in Ft Lauderdale, FL, USA Booth \#1312 August 5th-9th

\section{SEM Tech Solutions}

\section{Innovative \& New}

\section{3-D Roughness Analyzer}

(1 nm vertical resolution!)

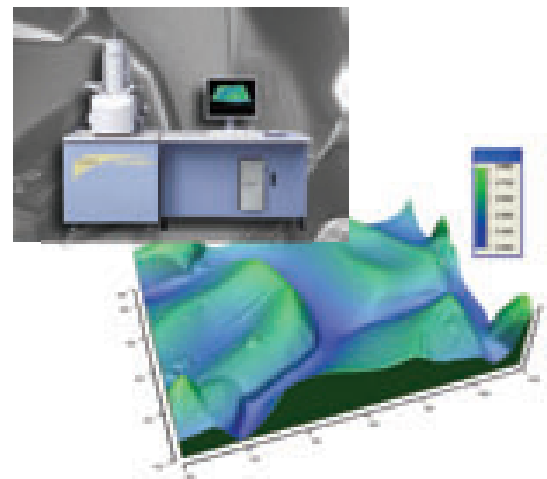

"Quickly move, image, zoom, and analyze in 3-D"
Refurbished

Factory Certified SEMs

(FE, $\mathrm{LaB}_{6}$, or $\mathrm{W}$ )

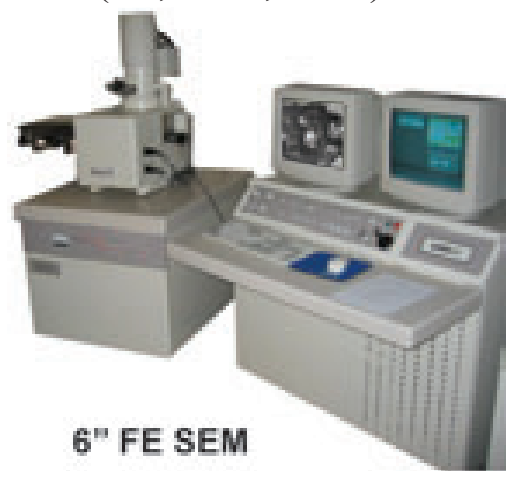

"Ask about our full service contract and leasing programs"

Used

Various SEM Models

(semtechsolutions.com/CurrentSemList.pdf)

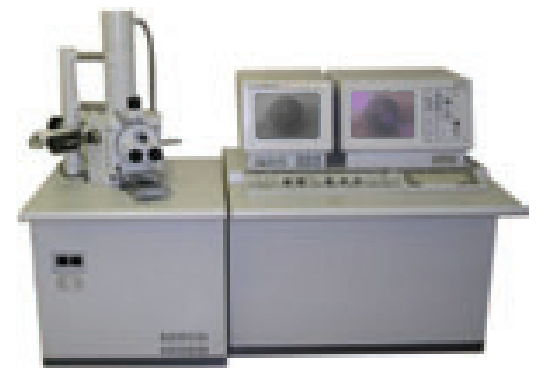

Hitachi S-2460N

Variable Pressure SEM

"Interested in a hard to get model not on our list? Call us!" 

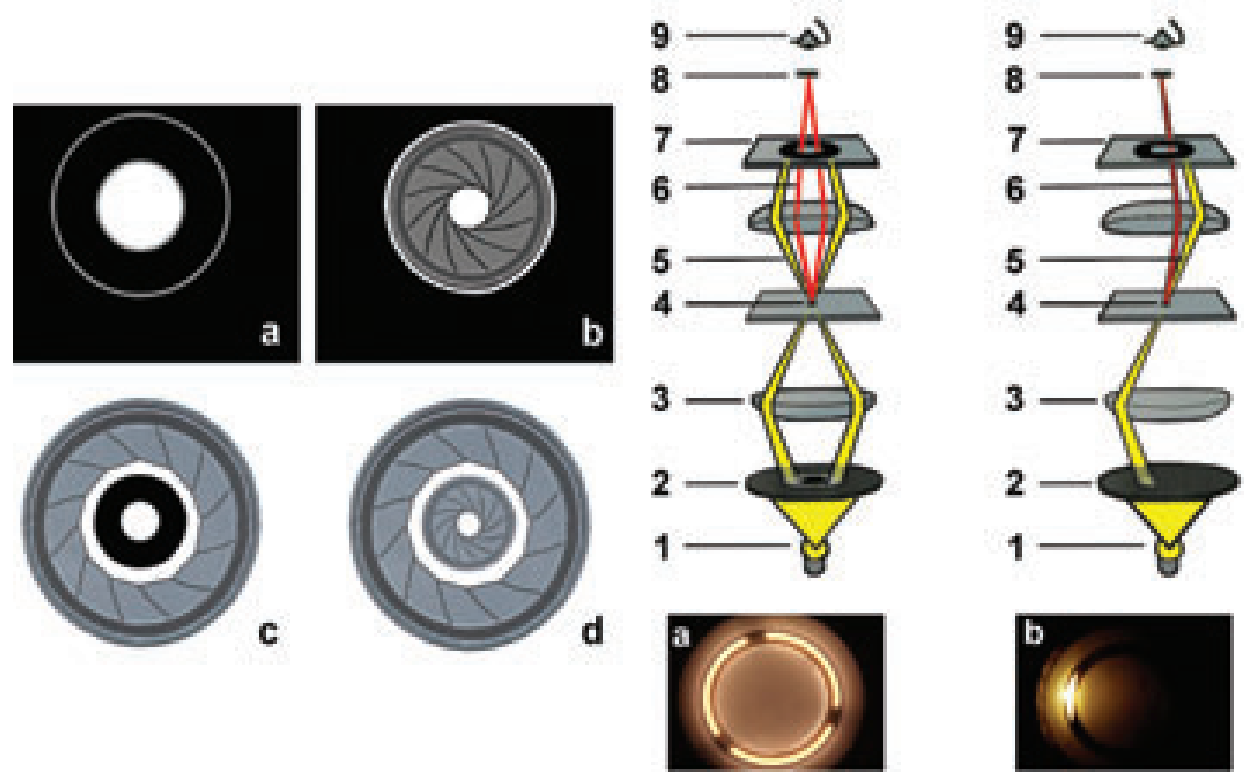

Fig. 27: Suggestions for various double diaghragm constructions. a: double diaphragm with fixed elements. $b$ : double diaphragm, fixed peripheral diaphragm, central iris diaphragm. $c$ : double diaphragm, fixed central diaphragm, peripheral iris diaphragm. d: double diaphragm, two concentric iris diaphragms

Fig. 28: Suggestions for modified constructions with annular light absorbers (modified from 7). a: luminace dark field in concentric illumination. b: luminance dark field in excentric unidirectional illumination. Key: 1 = light source; 2 = light ring; $3=$ condenser; $4=$ specimen; $5=$ illuminating light; $6=$ light bent by the specimen; $7=$ annular beam stop, $8=$ eyepiece with intermediate image; $9=$ eye

Fig. 29: Achievement of variable light rings in condensers. a: construction plan, central absorber plate, peripheral iris diaphragm. $b$ : transparent filter for bright field condensers, centric light absorber. c: alignment of the light absorber, controlled by a phase telescope, aperture iris diaphragm in wide position. $d$ : aperture diaphragm in intermediate position. e: aperture diaphragm in subtotally closed position

Moreover, the paracentral light beams within the objective, which are also important for microscopic images are seriously compromised instead of the central light components. This manipulation of the light path could also lead to additional visible degradation.

\section{Discussion}

Luminance contrast, presented as a new illumination technique, has been developed based on hand-made prototypes of objectives and light modulating elements. The conventional lenses, which were equipped with special light absorbing platelets as well as the Cassegrain-Schwarzschild mirror objectives had been fabricated some decades ago. Nevertheless, the luminance contrast images resulting from these optical components are characterized by extraordinary contrast, excellent sharpness and supramicroscopic resolution. In all objectives, the image quality was visibly improved when compared with conventional illuminating modes carried out using the same components. Therefore, this new method promises further fundamental improvements of quality when modern objectives are modified for luminance contrast based on up-to-date manufacturing technologies. Conventional lenses for phase contrast could easily be modified for luminance contrast by replacing their phase rings with a centered beam stop. Alternatively, a beam stop could be mounted instead of a phase ring according to the construction plan shown in fig. 6 or carried out in variant constructions, e.g. mounted only with one single or two opposite arms. Mirror objectives might be manufactured with improved precision and quality utilizing modern manufacturing methods. In luminance contrast, mirror systems might be an advantage, because their construction is a priori based on a centric mirror that can act as a beam stop. The complete absence of chromatic aberration and the planarity of great visual fields may be discussed as additional advantages.

In luminance contrast, the various effects of illumination can be achieved and changed in a simple manner, when the diameter of the aperture diaphragm is appropriately adjusted. Thus, the image can be turned from luminance dark field to luminance phase contrast, when the aperture diaphragm is opened moderately. In the same way, the intensities of contrast and background illumination can be regulated in tiny steps and halo artifacts can also be avoided, if necessary. In luminance dark field, marginal blooming effects are less than in the conventional mode. As all illuminating light beams are centered and running in an axial direction, many more details inside the specimen can be detected. The illumination of specimens is much more homogeneous than in conventional dark field, thus, specimens are seen as self luminous or florescent bodies. Probably because of this reason, very small structures can be visible in luminance contrast that are smaller than the usual resolving power of the respective optical system. In this respect, luminance contrast might be comparable to fluorescence techniques. Further improvements can be expected, when the various modes of technical developments are implemented described above. Thus, luminance contrast might also be capable to give new improving impulses for fluorescence microscopy.

\section{References:}

(1) Castro-Ramos, J., Cordero-Davila, A., Vazquez-Montiel, S., Gale, D.: Exact design of aplanatic microscope objectives consisting of two conic mirrors, Appl. Opt. 37, 5193-5197, 1998

(2) Davin Optronics: Reflecting microscope objectives, 2007, http://www.gmp.ch/index.php?main_page=product_info\&manufacturers_id=32\&products_id=215

(3) Determann, H., Lepusch, F.: The light microscope and its use (in German), Wild Leitz, Wetzlar, 1988

(4) Ernst Leitz Wetzlar GmbH: The imaging and illuminating optical elements in light microscopes (in German), Druckschrift, Wetzlar, 1969

(5) Gehne, H.: Mirror objectives for light microscopes (in German), Physikalische Blätter, 8. Jahrg., Heft 10, 453-460, Physik Verlag, Mosbach/Baden, 1952

(6) Jenoptik Jena GmbH: Mirror objectives and mirror condensers for microscopic examinations in ultraviolet, visible and ultrared light spectra, technical description (in German), Jena, 1967

(7) Nobel Foundation: The phase contrast microscope, http://nobelprize.org/educational_games/physics/microscopes/phase/index.html

\section{Acknowledgements:}

The author thanks Prof. Dr. Klaus Hausmann and Dr. Renate Radek, Free University of Berlin, for pure cultures of several protozoa and Timm Piper for the image shown in Figure 13. 


\section{Conquering the sub Ångstrom era}

Ultimate performance, stability, and flexibility-FEI's revolutionary Titan is the first and only platform designed to realize the full potential of corrected S/TEM imaging and analysis. With directly interpretable, sub Ångstrom image resolution you will, quite literally, see things you've never seen before. Investigate property/structure relationships with atomic resolution. Clearly visualize the interfaces, discontinuities and crystalline defects that are often the key to material properties. Analyze elemental composition, chemical bonding states, electronic band gaps, and more with unprecedented spatial resolution. If you know S/TEM, you know this truly is a revolution.

If your research requires ultimate performance, and your success depends on generating ground breaking new results, call us, we'll be happy to give you an unfair advantage.

See more at fei.com 